\title{
Small-mass Neutrinos, Massless Neutrinos, and Gauge Transformations
}

\section{M.E. NOZ*}

New York University

E-mail: marilyne.noz@gmail.com

\section{Y.S. KIM}

University of Maryland

E-mail: yskimeumd.edu

\begin{abstract}
Neutrino oscillation experiments presently suggest that neutrinos have a small but finite mass. This paper shows how the Dirac equation can be used to distinguish between massive and massless particles. In the large-momentum/zero mass limit, the Dirac spinors can be separated into large and small components. The large components are gauge invariant, while the small components are not. If we renormalize the large components, these gauge invariant spinors represent the polarization of neutrinos. The small components represent spin- $\frac{1}{2}$ non-zero mass particles. A similar analysis is then done when the neutrino mass is considered as an invariant of the Lorentz transformation. If neutrinos are to have mass, then they should not be invariant under gauge transformations.
\end{abstract}

The European Physical Society Conference on High Energy Physics 18-24 July, 2013

Stockholm, Sweden

\footnotetext{
* Speaker.
} 


\section{Dirac Spinors and Massless Particles}

In his 1939 paper [1], Wigner proposed that the symmetry properties of free particles be studied by using operators which commute with the specified four-momentum of the particle. He defined the "little groups" to be those transformations that do not change this four-momentum. For massive particles, the little groups are isomorphic to $\mathrm{O}(3)$ and for massless particles they are isomorphic to $\mathrm{E}(2)$.

To apply the Dirac equation to spin- $\frac{1}{2}$ particles, we consider the group generated by:

$$
S_{i}=(1 / 2) \sigma_{i}, \text { and } K_{i}=(i / 2) \sigma_{i}
$$

Here the $\sigma_{i}$ are the Pauli matrices and the $\mathrm{S}$ operators represent rotations while the $\mathrm{K}$ operators represent boosts.

Starting with these spin operators, a boost along the $\mathrm{z}$ direction will take the form:

$$
S_{i}^{\prime}=B(P) S_{i} B^{-1}(P)
$$

Here the boost matrix is given by:

$$
B(P)=\left(\begin{array}{cc}
e^{\xi / 2} & 0 \\
0 & e^{-\xi / 2}
\end{array}\right) .
$$

In the large-momentum limit for a massive particle we obtain:,

$$
e^{\xi} \rightarrow \frac{2 E}{M}
$$

The gauge transformation matrices take the form $[2,3]$

$$
D(u, v)=\left(\begin{array}{cc}
D^{(+)}(u, v) & 0 \\
0 & D^{(-)}(u, v)
\end{array}\right) .
$$

This matrix is applicable to the Dirac spinors. If the spinors representing a massive particle at rest are boosted along the z-axis using the operator generated by $K_{3}$, then

$$
U(P)=\left(\begin{array}{c}
e^{(+\xi / 2)} \alpha \\
\pm e^{(-\xi / 2)} \dot{\alpha}
\end{array}\right), \quad V(P)=\left(\begin{array}{c} 
\pm e^{(-\xi / 2)} \beta \\
e^{(+\xi / 2)} \dot{\beta}
\end{array}\right)
$$

In the large-momentum/zero mass limit, the large components, $e^{(+\xi / 2)}$, are gauge invariant, while the small components are gauge dependent. This shows that non-zero mass, spin- $\frac{1}{2}$ particles are not invariant under gauge transformations. Furthermore, the spinors of Eq.(1.6) can be renormalized but are still invariant under the $D$ transformation. It is this invariance that is responsible for the polarization of neutrinos $[2,3]$.

Additionally, interpreting the results of Eq.(1.6) in terms of E(2) translations on free Weyl neutrino states, the gauge invariant transformations leave the left-handed neutrino invariant, but translate the right-handed neutrino into a linear combination of left-handed and right-handed neutrinos $[2,4]$. 


\section{Neutrino Mass and Lorentz Transformations}

Because there is no Lorentz frame in which a massless particle is at rest, we consider a momentum four-vector of the form:

$$
P=(0,0, \omega, \pm \omega)
$$

and begin with a massive particle with fixed energy $E$ boosted along the $z$ axis:

$$
z \rightarrow(\cosh \xi) z+(\sinh \xi) t, \quad t \rightarrow(\sinh \xi) z+(\cosh \xi) t .
$$

Within the framework of Lorentz transformations, $E$, and thus $\xi$, can become large [5].

In addition, $\xi$ can become large when the mass becomes very small. This cannot be achieved by Lorentz boosts, because the mass is a Lorentz-invariant quantity. With this point in mind, we can consider what happens when the mass is varied but the energy is held fixed. We can write the energy-momentum four-vector as

$$
E(0,0, \cos \chi, 1)
$$

Then the mass becomes

$$
M=E \sqrt{1-\cos ^{2} \chi}=E \sin \chi .
$$

Hence, the mass can be increased by increasing $\chi$ from zero [6]. The four-by-four matrix which makes the transformation of Eq.(2.2) has a two-by-two matrix equivalent to the spinor [6]. If we now perform the Lorentz boost by making a similarity transformation, then in order for the matrix to remain finite in the large $\xi$ limit, we can let $e^{\xi} \sin \theta=\gamma$. For $\gamma$ to remain finite as $\xi$ increases, $\theta$ must approach zero. Thus, the matrix becomes

$$
\left(\begin{array}{cc}
1 & -\gamma \\
0 & 1
\end{array}\right)
$$

It has been shown that the $\gamma$ parameter performs gauge transformations on the photon case, and its equivalent transformation on massless neutrinos [5, 2]. If the neutrino indeed has mass, then we should observe the neutrinos participating in gauge transformations.

\section{References}

[1] E. Wigner, On unitary representations of the inhomogeneous lorentz group, Annals of Mathematics 40 (149) 1939.

[2] D. Han, Y. Kim, and D. Son. E(2)-like little group for massless particles and neutrino polarization as a consequence of gauge invariance, Physical Review D, 26 (3717) 1982. doi:10.1103/PhysRevD.26.3717.

[3] Y.S. Kim, Neutrino polarization as a consequence of gauge invariance, Czechoslovak Journal of Physics 52(S3) (C353) 2002. doi:10.1007/s10582-002-0129-6.

[4] A.O. Barut and J. McEwan, The four states of the massless neutrino with pauli coupling by spin-gauge invariance, Letters in Mathematical Physics 11 (67) 1986. doi:10.1007/BF00417466.

[5] Y.S. Kim and M.E. Noz, Theory and applications of the PoincareİA group, Fundamental theories of physics. Reidel ; Sold and distributed in the U.S.A. and Canada by Kluwer Academic Publishers, Dordrecht, Holland ; Boston : Hingham, MA, 1986.

[6] Y.S. Kim and M.E. Noz, Symmetries shared by the poincaré group and the poincaré sphere, Symmetry 5 (233) 2013. doi:10.3390/sym5030233. 